\title{
Influence of Breastfeeding Promotion Package on Breastfeeding Practices among Women- A Cohort Study
}

\author{
Ariff $S^{1}$, Tariq $B^{2}$, Sadiq $K^{1}$, Sikanderali $L^{1}$, Shaheen $\mathrm{F}^{1}$, Aamir $\mathrm{A}^{1}$, Rizvi $\mathrm{A}^{1}$, Ali $\mathrm{AA}^{1}$, Habib MA ${ }^{* 1}$ and \\ Soofi $\mathrm{SB}^{1}$
}

${ }^{1}$ Department of Pediatrics and Child Health, Aga Khan University, Karachi, Pakistan

'Ziauddin University, Karachi, Pakistan

${ }^{*}$ Corresponding author: Habib MA, Assistance Professor, Department of Pediatrics and Child Health, Aga Khan University, Karachi, Pakistan, Tel: +923002769398, E-mail: atif.habib@aku.edu

Citation: Ariff S, Tariq B, Sadiq K, Sikanderali L, Shaheen F, et al. (2020) Influence of Breastfeeding Promotion Package on Breastfeeding Practices among Women- A Cohort Study. J Paedatr Neonatal Dis 5(1): 103. doi: $10.15744 / 2456-5482.5 .103$

Received Date: November 03, 2020 Accepted Date: December 02, 2020 Published Date: December 04, 2020

\begin{abstract}
Background: Exclusive breastfeeding is essential for the first 6 months of life. In Pakistan, the proportion of children under age 6 months who are exclusively breastfed is only 38 percent making it urgent to explore interventions to address the low rates.

Research Aim: To investigate the influence of breastfeeding promotion package on breastfeeding practices during the first 6 months of life.

Methods: We conducted a quasi-experimental study. The intervention group received a promotion package with educational leaflets, health education and telephonic counseling. The control group received routine breastfeeding counseling. Baseline information was recorded at enrollment with follow-up till the age of 6 months. Intervention and control groups were compared using ANOVA and chi-square tests for mean and percentage differences, respectively.
\end{abstract}

Results: 250 participants were recruited in each group. 30\% of mothers exclusively breastfed their child at six months of age in the intervention group, as compared to only $12.6 \%$ in the control group $(\mathrm{P}<0.0001)$. Infant's age at introduction of mixed feeding was also found to be significantly different between the two groups. Multiparous women, age $>35$ and home-makers were more likely to exclusively breastfeed. Reductions in lower respiratory tract infection (3\%), diarrhea (6\%), upper respiratory infection (4\%), and skin lesions (5\%) were seen in the intervention group.

Conclusion: Our study identifies barriers to exclusive breastfeeding and provides evidence that education, one-to-one, telephonic counseling, and early post-natal visit can improve breastfeeding rates. Results of this study should inform future programs aimed at improving breastfeeding rates at local and government levels.

Keywords: Anophthalmia; Arrhinia; Choanal Atresia; Hypogonadotrophic Hypogonadism, Microphthalmia

\section{Background}

Neonatal mortality is an important global public health problem especially in developing countries. The neonatal mortality rate is reported as 46 per 1000 live births and the under-five mortality rate as 81 per 1000 live births in Pakistan ((UNICEF., United Nations population., \& Bank), 2015) [1]. Main causes leading to neonatal deaths include prematurity (39\%), birth asphyxia and trauma $(21 \%)$ and sepsis $(17 \%)$ (WHO, 2015) [2]. Globally, one third of under-five mortality is due to malnutrition, of which two third die during the first year of life. Interventions promoting optimal breastfeeding could prevent $13 \%$ of deaths in countries with high mortality rates (Kimani-Murage et al., 2011) [3]. When a child is exclusively breastfed, the immune system is strengthened, enabling it to combat life threatening illnesses like pneumonia, skin infections and diarrhea (Hanieh et al., 2015) [4]. Breastfed children have at least six times greater chance of survival in the early months than non-breastfed children (Cai, Wardlaw, \& Brown, 2012) [5]. Moreover, past studies have found correlation between breastfeeding and reduced frequency of respiratory and gastrointestinal infections (Frank et al., 2019; Horta, Bahl, Martinés, Victora, \& WHO, 2007) [6,7]. Breastfeeding is also associated with reduced risk of non-communicable diseases such as obesity, hypertension and diabetes (Horta et al., 2007; Horta \& Victora, 2013) [7,8].

United Nations International Children's Emergency Fund UNICEF reported that exclusive breastfeeding (EBF) practices in Pakistan were 37\% in 2006-2007 (Jamil, Khanum, Omer, \& Hamid, 2018) [9], however the recent data on exclusive breastfeeding is encouraging and $48 \%$ of mothers reported exclusive breastfeeding in the National Nutrition Survey (NNS) 2018 and Pakistan 
Demographic and Health Survey (PDHS) (NIPS \& ICF International, 2019; UNICEF, 2019) [10,11]. The results in both surveys showed an evident increase in exclusive breastfeeding over the past 5 years when $38 \%$ of children under 6 months were exclusively breastfed. Various socioeconomic and demographic factors are identified as a cause of low exclusive breastfeeding practices in Pakistan. These factors include poverty, illiteracy, gender of the infant (Heil et al., 2017) [12], lack of knowledge about (Farrukh, Basheer, \& Jalil, 2013) [13] the benefits of EBF and personal perceptions of the family and the mother herself (Heil et al., 2017) [12]. Furthermore, early introduction of complementary foods in Pakistan is a significant factor contributing towards low rates of exclusive breastfeeding. Almost $8 \%$ of infants under the age of 6 months consume plain water, 23\% other milk and $13 \%$ receive complementary foods (NIPS \& ICF International, 2019) [14].

We carried out a quasi-experimental study at a tertiary care hospital in Karachi to evaluate interventions for improvement in the current low rates of breast feeding. Our intervention was introduction of a comprehensive "breastfeeding promotion package" for mothers to reinforce the importance of exclusive breastfeeding in infants. The aims of this study were to: 1) Evaluate the influence of breastfeeding promotion package on breastfeeding practices during the first 6 months of life 2) Identify the factors affecting exclusive breastfeeding rates 3) Assess the effect of breastfeeding on infant morbidity.

\section{Methodology}

\section{Study Setting}

This study was conducted as a nested study within the Multi-center body composition reference study (MBCRS) at a tertiary care hospital from October 2014 till April 2016. The aim of MBCRS was to produce body composition reference data in healthy children across different ethnicities. The hospital is a tertiary care referral facility that provides essential maternal and newborn care along with standard counseling as part of routine to ensure EBF practices.

\section{Study Design}

Our study design was quasi-experimental. The institution review board and ethics review committee of the hospital approved the study.

\section{Sample}

The inclusion criteria for the original (MBCRS) study were; mothers of age 18 years and above, giving birth to term babies, having an intention to exclusive breastfeed for 6 months, completed their secondary level of education, non-smokers and living in close proximity of the hospital. The control group comprised of pregnant women who received antenatal care and delivered at the hospital. All other eligibility criteria were identical to the intervention group. As part of Baby Friendly Hospital, mothers in the control group received routine advice and recommendations on breastfeeding practices from health care providers during hospital stay. This includes optional antenatal classes on breastfeeding and counseling by midwives and nurses in the post-natal wards. The optimum sample size of 250 study participants was calculated on the basis of $38 \%$ exclusive breastfeeding rate according to demographic and health survey of Pakistan (2012 -2013) with 90\% confidence interval and 5\% limit of error (NIPS \& ICF International, 2013) [14]. Participants in the intervention group were originally enrolled in the larger MBCRS.

Breastfeeding Promotion Package: A comprehensive intervention package was developed for the mothers in addition to the regular standard counseling routinely provided to ensure EBF practices. The package included; educational leaflets, health education session, telephonic counseling.

Educational Leaflets: Educational leaflets with information on breastfeeding practices were provided to the mothers at delivery, 3 months and subsequent post-natal follow up visit. Information on the benefits of breastfeeding, methods of breast feeding, nutritional requirements of lactating mothers, and potential problems encountered during breast feeding were explained in simple language. The leaflet was available both in English and local language.

Health Education Session by Nurses and Midwives: Breastfeeding sessions in groups were conducted for both the parents, on quarterly basis. Parents were invited to attend the sessions, however only mothers participated, and majority attended at least one session. The sessions were facilitated by a senior experienced staff with 15 years of experience in lactation management.

Telephonic Based Lactation Counseling: The third component was telephonic calls to the participants, on monthly basis for the first 6 months. Follow up on breastfeeding practices was documented; queries addressed, and appropriate advice given. Key messages conveyed during telephonic counseling are shown in Figure 1. Mothers, who did not respond to telephonic calls, were approached again for three consecutive days at different timings. They were also encouraged to call the hot line created for this purpose during working hours.

Post-Natal Clinic with Physician: Out-patient clinics appointments were arranged with Neonatologist at first post-natal contact and on quarterly basis. Breastfeeding techniques were reviewed by the nurse and female physician and mother's questions and concerns were addressed. During post-natal clinics, emphasis was given by the physician on; feeding on demand (8-12 times/day), advantages of breast milk and its protective impact on childhood morbidity. 


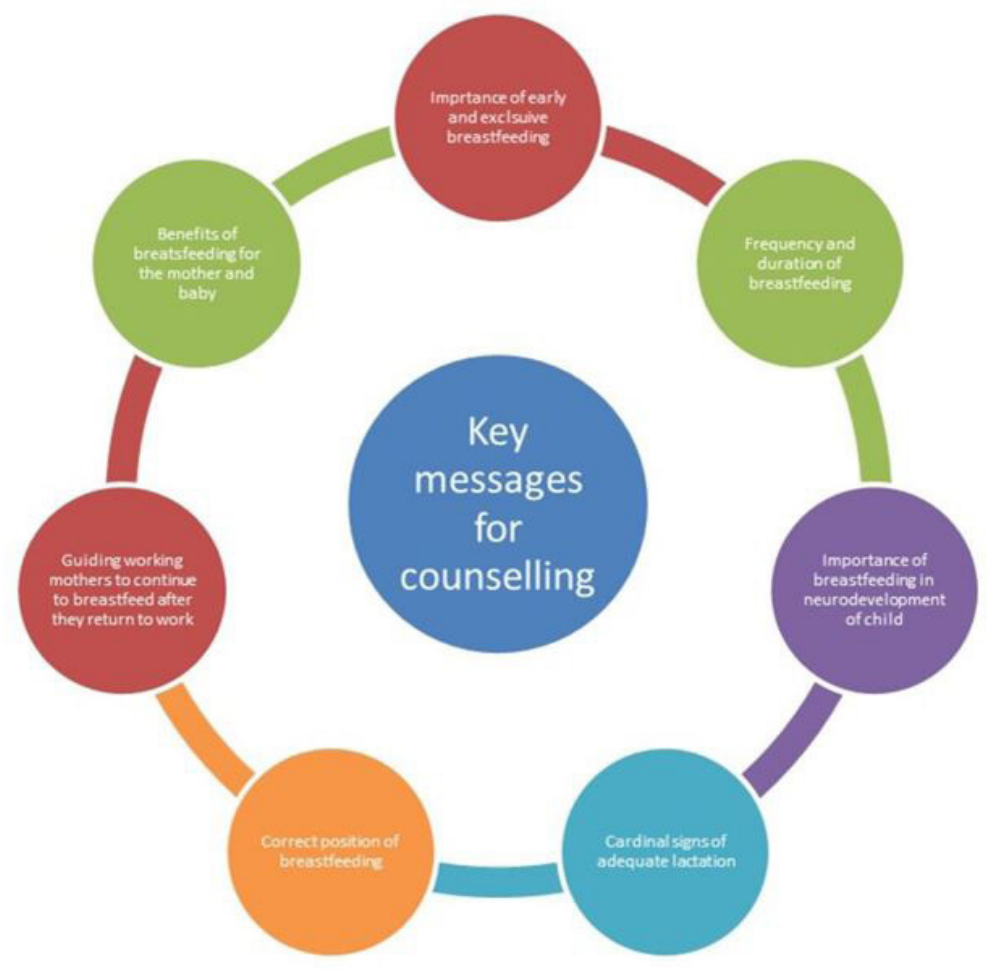

Figure 1: Key messages conveyed during telephonic counseling

\section{Data Collection}

The participants who met the inclusion criteria and signed the informed consent were included. Information on the mother and infant dyad was collected on a structured questionnaire. Baseline information on mothers (e.g., demographics, maternal age, number of children,) and their infants (e.g., gestational age, birth weight,) was recorded at the time of study enrollment. Follow-up data was collected till the age of 6 months. Data on feeding and morbidity was collected at monthly intervals from the mothers, as maternal recall. The calls were made at the end of each month for 6 consecutive months. Both mother-reported child illness and physician confirmed illness were included in the morbidity data. Breast feeding practices including compliance were documented with the help of a standard Performa that was filled at the end of the call.

Blinding of study participants was not feasible due to the nature of the intervention. However, the research assistant responsible for collecting information on breastfeeding via monthly phone calls was blinded to group allocation.

\section{Data Analysis}

Data was entered on screens developed in Visual Basic Net using SQL server 2008 database. All data was transferred and analyzed using SPSS (Statistical Package for Social Sciences, Version 15.0). Data was presented as mean and standard deviation (SD) in case of continuous variables and categorical variables presented as frequencies and percentages. Intervention and control groups were compared using ANOVA and chi-square tests for mean and percentage differences respectively. $\mathrm{P}$ value $<0.05$ was considered as statistically significant difference. Multivariate logistic regression analysis was applied to evaluate factors associated with exclusive breastfeeding.

\section{Ethics}

The study was approved by Aga Khan University Ethical Review Committee. ERC number: 3148-PED-ERC-14.

\section{Results}

250 participants contributed to each study group. The mean age of mothers across both groups was 29 years and on average, women completed 14 years of education (Table 1). 


\begin{tabular}{|c|c|c|c|}
\hline & $\begin{array}{c}\text { Intervention } \\
n=250\end{array}$ & $\begin{array}{l}\text { Control } \\
n=250\end{array}$ & Total \\
\hline Maternal Age (Years) mean \pm SD & $28.5 \pm 4.4$ & $29.4 \pm 4.8$ & $28.9 \pm 4.6$ \\
\hline$<25$ years $n(\%)$ & $174(69.6)$ & $152(60.8)$ & $326(65.2)$ \\
\hline $25-35$ years $n(\%)$ & $53(21.2)$ & $62(24.8)$ & $115(23)$ \\
\hline$>35$ years $n(\%)$ & $23(9.2)$ & $36(14.4)$ & $59(11.8)$ \\
\hline Maternal Education (Years) mean $\pm S D$ & $14.4 \pm 2.5$ & $14.3 \pm 2.8$ & $14.3 \pm 2.6$ \\
\hline Above bachelors $n(\%)$ & $124(49.6)$ & $158(63.2)$ & $282(56.4)$ \\
\hline Up to bachelors $\mathrm{n}(\%)$ & $126(50.4)$ & $92(36.8)$ & $218(43.6)$ \\
\hline Gestational Age (Weeks) mean \pm SD & $38.5 \pm 2.0$ & $38.2 \pm 1.03$ & $38.3 \pm 1.6$ \\
\hline \multicolumn{4}{|l|}{ Occupation $n(\%)$} \\
\hline Housewife & $201(80.4)$ & $135(78.95)$ & $336(79.81)$ \\
\hline Working mothers & 49 (19.6) & $36(21.05)$ & 85 (20.19) \\
\hline \multicolumn{4}{|l|}{ Monthly Income $n(\%)$} \\
\hline $50000-75000$ & $97(38.8)$ & $21(65.63)$ & $118(41.84)$ \\
\hline $75000-100000$ & $73(29.2)$ & $8(25)$ & $81(28.72)$ \\
\hline$>100000$ & $80(32)$ & $3(9.38)$ & $83(29.43)$ \\
\hline \multicolumn{4}{|l|}{ Infant's Gender $n(\%)$} \\
\hline Male & $131(52.4)$ & $119(47.6)$ & $250(50)$ \\
\hline Female & $119(47.6)$ & $131(52.4)$ & $250(50)$ \\
\hline Birth Weight $(\mathrm{kg})$ mean $\pm S D$ & $3.11 \pm 0.38$ & $2.96 \pm 0.45$ & $3.03 \pm 0.42$ \\
\hline LBW (birth weight $<2.5 \mathrm{~kg}$ ) & $11(4.4)$ & $24(9.6)$ & $35(7)$ \\
\hline
\end{tabular}

Note: Data presented as mean $\pm S D$ and $n(\%)$

Table 1: Characteristics of Maternal, Socio-demographic and Children Factors at Baseline

$80 \%$ of the mothers were homemakers in intervention group as compared to $78 \%$ in control group. The two groups were comparable as shown in Table 1. Follow up of the study participants is shown in Figure 2.

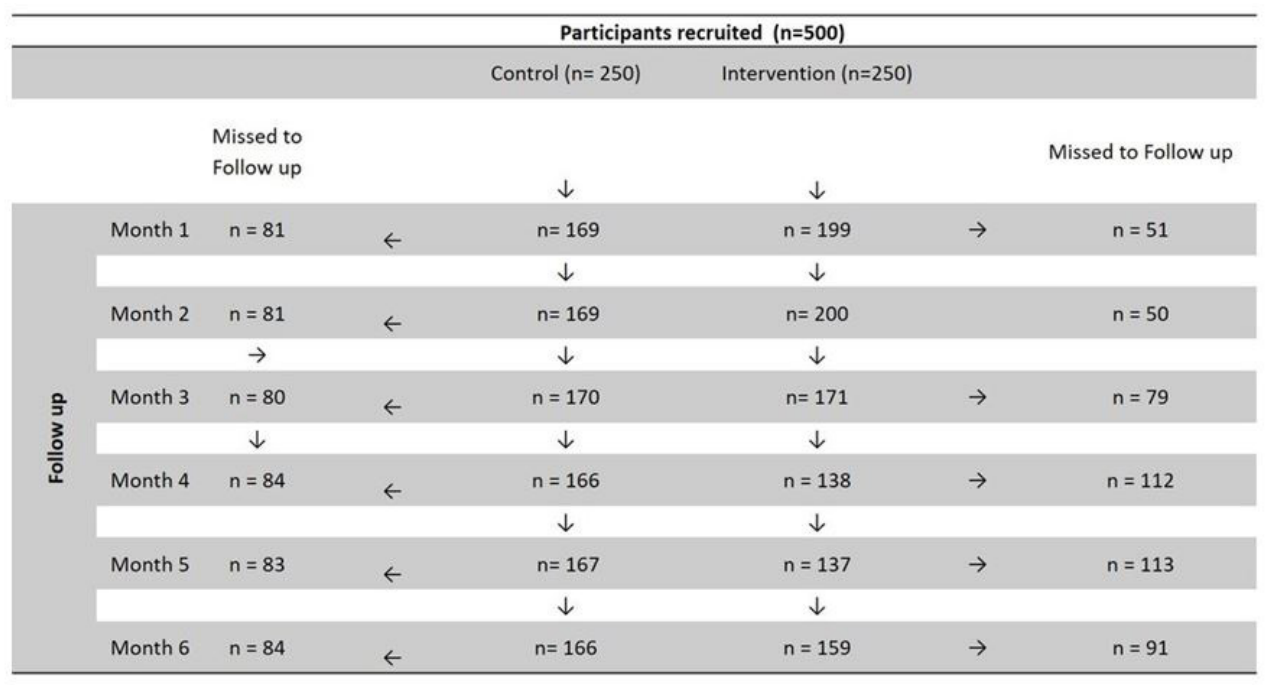

Figure 2: Follow up of the study participants

\begin{tabular}{|c|c|c|c|}
\hline & $\begin{array}{c}\text { Intervention } \\
\mathbf{n = 1 5 9}\end{array}$ & $\begin{array}{c}\text { Control } \\
\mathbf{n = 1 6 6}\end{array}$ & P-value \\
\hline & $n(\%)$ & $n(\%)$ & \\
\hline Proportion of infants exclusively breastfed & $48(30.2)$ & $21(12.6)$ & $<0.0001$ \\
\hline Proportion of infant fed by mix feeding & $111(69.8)$ & $145(87.3)$ & $<0.0001$ \\
\hline & Mean \pm SD & Mean \pm SD & \\
\hline Age till infant is exclusively breastfed & $3.64 \pm 1.55$ & $3.96 \pm 1.54$ & 0.096 \\
\hline Age at which mix feeding introduced & $4.09 \pm 2.45$ & $3.32 \pm 2.03$ & 0.002 \\
\hline
\end{tabular}

Note: Data presented as mean \pm SD and $n(\%)$

Table 2: Breastfeeding Status at 6 Months 
Analysis of the data identified important differences in breastfeeding practices between the two groups (Table 2). Out of 250 Mothers in the intervention group, 30\% exclusively breastfed their infants till 6 months whereas, $69 \%$ practiced mix feeding, in comparison to the control group, where only $12 \%$ mothers exclusively breast fed (Table 2 ).

A significant difference was noted between the ages at which mix feeding was introduced, across the two groups, (4 months vs 3 months, $\mathrm{P}=0.002)$. Furthermore, as shown in Figure 3, the number of women who exclusively breastfed (EBF) were found to be high in the intervention group, as compared to the control group, especially during the first four months. One of the reasons for the difference between the two groups could be due to the one-to-one counseling provided to the mothers in the intervention group where their queries were addressed. Some of the issues faced by the lactating mothers were about the perception of insufficient milk, switching from one breast to another and regarding breastfeeding during mother's illness.

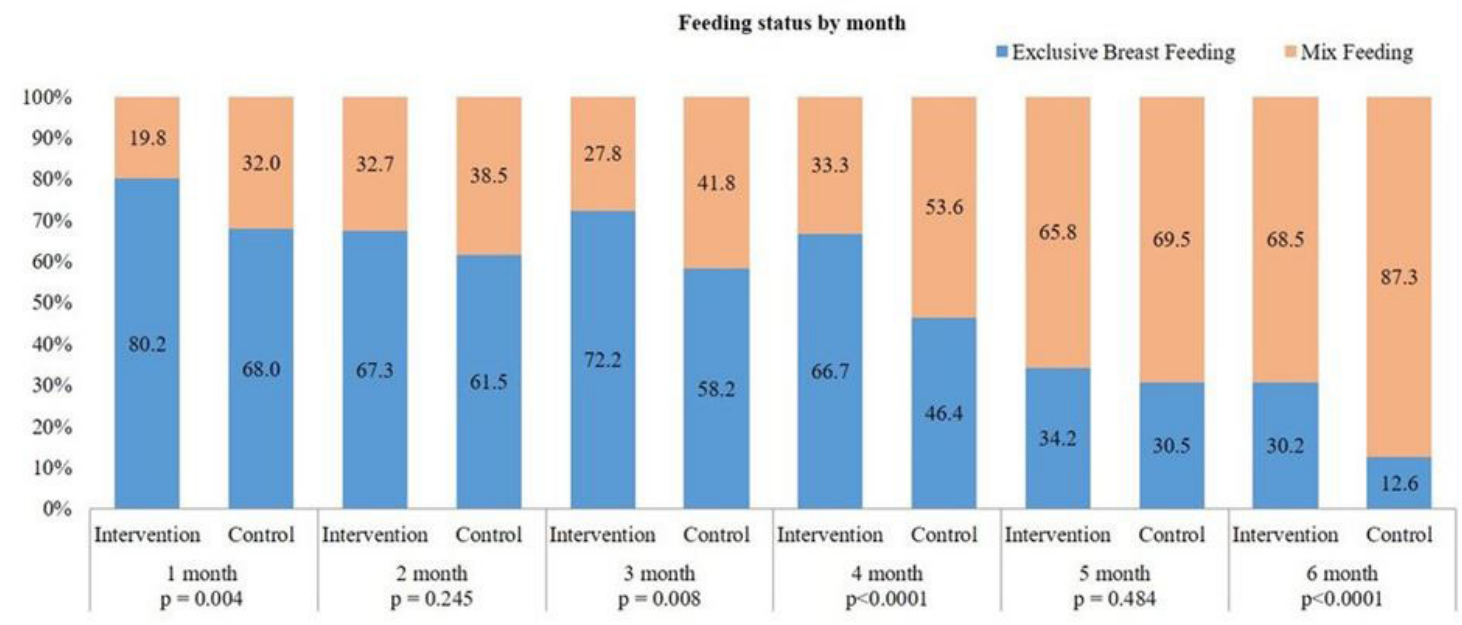

Figure 3: Significant difference was noted between the ages at which mix feeding

The gender of the infant, maternal age, education or occupation had no significant association with infants who weren't exclusively breastfed. However, parity had a significant association with non-exclusive breast feeding $(\mathrm{P}=0.001)$ indicating multiparous mothers are more likely to exclusively breastfed their babies as compare to primiparous mothers (Table 3 ). This identifies first time mothers as a high-risk group (because of their lack of experience) and therefore should be offered additional support, especially practical help, in order to overcome breastfeeding challenges.

Women with maternal age $>35$ years were more likely to exclusively breastfeed compared with $<35$ years old even though no significant association was noted between mother's occupation and EBF, but homemakers were more likely to exclusively breast feed as compared to working mothers (Table 3).

\begin{tabular}{|c|c|c|c|}
\hline & \multicolumn{3}{|c|}{ Multivariate Model } \\
\hline & $\mathbf{R R}$ & $95 \% \mathrm{CI}$ & P-value \\
\hline \multicolumn{4}{|l|}{ Group } \\
\hline Control & 2.93 & $2.03,4.23$ & $<0.0001$ \\
\hline \multicolumn{4}{|l|}{ Maternal age (Years) } \\
\hline$<25$ years & Ref. & & \\
\hline 25-35 years & 0.96 & $0.52,1.78$ & 0.904 \\
\hline$>35$ years & 1.26 & $0.65,2.43$ & 0.493 \\
\hline \multicolumn{4}{|l|}{ Maternal education (Years) } \\
\hline Bachelors and above & 1.00 & $0.65,1.54$ & 0.989 \\
\hline Up to bachelors & Ref. & & \\
\hline \multicolumn{4}{|l|}{ Occupation } \\
\hline Housewife & Ref. & & \\
\hline Working mothers & 1.12 & $0.82,1.52$ & 0.479 \\
\hline \multicolumn{4}{|l|}{ Monthly income } \\
\hline $50000-75000$ & Ref. & & \\
\hline $75000-100000$ & 0.8 & $0.54,1.19$ & 0.272 \\
\hline$>100000$ & 1.07 & $0.75,1.52$ & 0.703 \\
\hline
\end{tabular}




\begin{tabular}{|c|c|c|c|}
\hline & \multicolumn{3}{|c|}{ Multivariate Model } \\
\hline & RR & $\mathbf{9 5 \%}$ CI & P-value \\
\hline No. of previous live births & & & \\
\hline First child & 3.17 & $1.65,6.06$ & 0.001 \\
\hline$\geq 1$ child & Ref. & & \\
\hline Gender & & & \\
\hline Male & Ref. & & \\
\hline Female & 1.14 & $0.83,1.57$ & 0.422 \\
\hline Birth weight $(\mathrm{kg})$ & & & \\
\hline NBW $\geq 2.5 \mathrm{~kg}$ & Ref. & & \\
\hline LBW $<2.5 \mathrm{~kb}$ & 0.99 & $0.42,2.31$ & 0.973 \\
\hline Co-morbidity & & & \\
\hline Yes & Ref. & & \\
\hline No & 0.71 & $0.48,1.04$ & 0.081 \\
\hline
\end{tabular}

Data presented in the form of RR=Relative Risk and 95\% Confidence interval

The analysis was done using GLM for binomial family

Table 3: Factors Associated with Non-Exclusive Breastfeeding at 6 months

We observed low rates of illnesses in the EBF group as compared to non EBF (Figure 4). There was a comparatively reduced rate of lower respiratory tract infection by $3 \%$, diarrhea by $6 \%$, upper respiratory infection by $4 \%$, and skin lesions by $5 \%$.

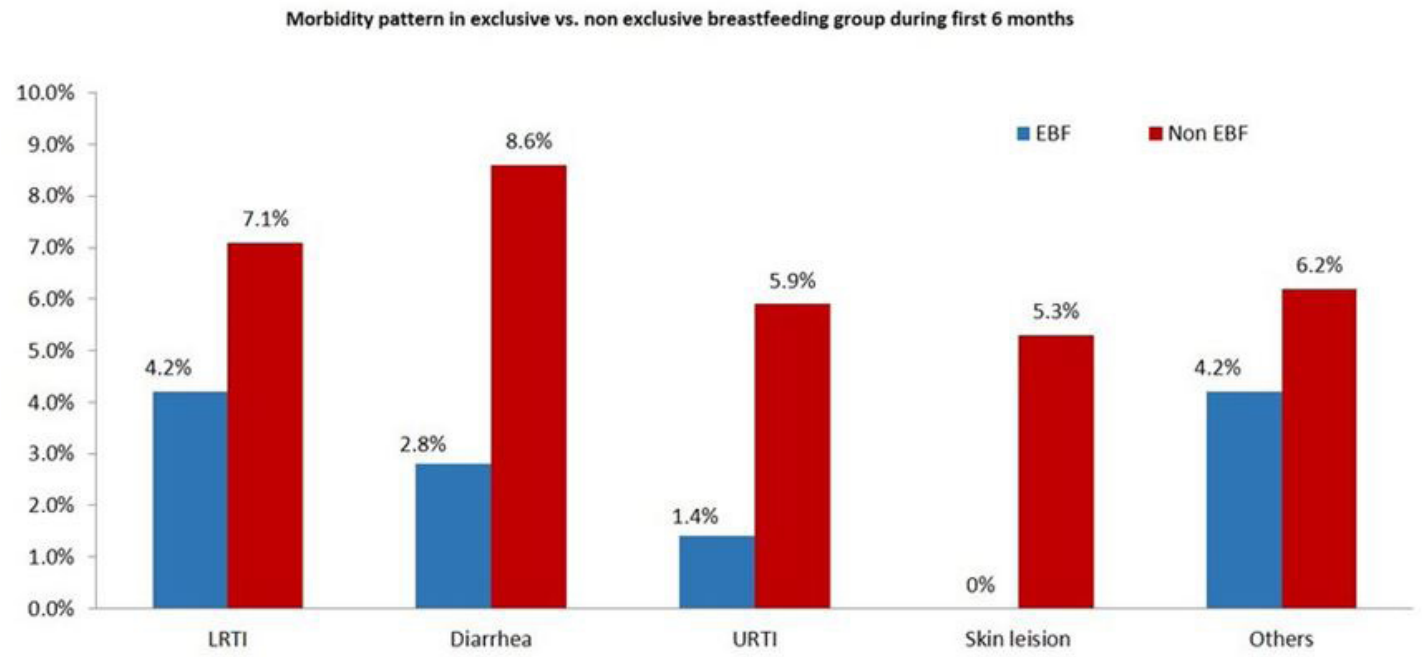

Figure 4: Rates of illnesses in the EBF group as compared to non EBF

\section{Discussion}

Exclusive breastfeeding is an ideal source of nutrition and energy for infants especially during the first six months of age. Yet only $30 \%$ of mothers practiced it following the introduction of intervention package.

Low rates of exclusive breastfeeding may largely owe to misconceptions and believes regarding insufficient milk production among mothers, compelling them to start complementary feeding or add formula to the diet. This was illustrated by the $60 \%$ of mothers who transitioned to mix feed after 3 months of age. The misconception prevails largely because majority of mothers particularly primi mothers are unaware of the proper technique and the benefits of breastfeeding as supported by our findings of multiparous more likely to EBF compared to primiparous.

The intervention package provided information about correct position for breastfeeding, cardinal signs of lactation and also reinforced on the benefits of breastfeeding that led to a significant difference between the two groups. EBF in the controls was only $12 \%$. Other beliefs that prevail in society are seasonal variation in breastfeeding practices. This relationship has been shown in prior studies as well. Infants born during summer months were exclusively breastfed for shorter duration compared to those born during winter season. Possible reasons cited were inadequate maternal nutrition during the pre-harvesting season of summers and mothers' concern regarding hydration status of their infants resulting in supplementation with liquid or semi-solid foods in the rural population (Velusamy, Premkumar, \& Kang, 2017) [18].

In Upper Sindh it is a cultural practice to EBF for 6 months in winters and less in summers (Bhutta, Soofi, Zaidi, \& Habib, 2011) [19]. Such beliefs are prevalent in regions where literacy rates are low (60\%) (NIPS \& ICF International, 2013) [14]. 
Our study also revealed that multiparous were more likely to EBF compared to primiparous primarily because of their experience and greater knowledge. This finding is supported by others (Hackman, Schaefer, Beiler, Rose, \& Paul, 2015) [20] where 35\% of primiparous women faced breastfeeding problems compared to $20 \%$ in multiparous women. The study also revealed that housewives were more likely to EBF as compared to working mothers, however this finding was not statistically significant. Previous studies have shown that mothers with full time employment are less likely to initiate breastfeeding or discontinue breastfeeding earlier than unemployed mothers (Lee, Wong, Lui, Chan, \& Lau, 2007; Tadesse, Alemayehu, Shine, Asresahegn, \& Tadesse, 2019) [21,22] due to several reasons including the lack of dedicated breastfeeding rooms at workplaces, a clean and hygienic environment. Lack of awareness on use of expressed mother's milk, manual as well as practice of electronic pump and encouragement by family and colleagues also play an important role (Tsai, 2013) [23]. A qualitative study from Pakistan showed that absence of support in the workplace hindered continuation of breastfeeding by working women (Hirani \& Karmaliani, 2013b) [24]. Interventions including childcare facilities, breast pumps and storage facilities, mother-friendly policies and provision of breastfeeding breaks were likely to enable sustained breastfeeding practices among working mothers (Hirani \& Karmaliani, 2013) [25].

This study attempts to provide evidence that, a multipronged approach consisting of health education, one to one counseling, telephonic contact, early post-natal visit and group sessions combined can improve the rates of EBF. A similar study showed that telephonic lactation counseling provided by certified lactation counselors (CLCs) was effective in increasing the rate of exclusive breastfeeding for the first postpartum month but not during the 4- and 6-month postpartum intervals (Tahir \& Al-Sadat, 2013) [26]. Both telephone and mobile phones are acceptable, cheap and accessible media of communication that are widely used. Thus, mothers who are usually 'housebound' in the early phase of the postnatal period may be better approached through telephone counseling. However, the results were not sustained and were unlikely related to the intervention (duration and number of successful calls) but possibly due to a single intervention emphasizing the importance of holistic approach and interventions.

We observed a high dropout rate in the intervention group (Figure 2). It is unlikely that the drop outs were related to the outcome being measured. Rather, many families found procedures of the main MBCRS study cumbersome. The larger study required administration of deuterium, saliva collection and significant stay at the clinical trial unit. Few families had to travel from distance and because of inconvenience decided to drop out.

We observed that due to lack of favorable environment at work place, mothers were unable to continue exclusive breastfeeding on resuming work. Majority of mothers were unaware of the use of expressed mother's milk and storage methods. This study highlights barriers to EBF that require attention from the government and local bodies. Our study highlights the significance of sustained and multipronged approach to improve exclusive breastfeeding rates. It also identifies major areas that require special attention such as additional support and motivation for first time mothers. Majority of interventions implemented in our breastfeeding promotion package were cost effective hence this package can serve in resource limited setting.

\section{Limitations}

This was a nested study and we were time bound; hence could not explore and expand on indirect determinants of breastfeeding trends. The response of the mothers depended upon how receptive they were to the counselor. We attempted to look into the relationship between the number of interventions and outcome however the numbers were not sufficient to achieve meaningful results. A larger sample size could be used to assess the efficacy of telephone-based counseling separately since this was a nested study within the original MBCRS study, we were unable to conduct and conclude on it. We were also unable to evaluate the impact of robust antenatal counseling session on breast feeding which has been shown to have a significant impact on early and exclusive breastfeeding in primiparous women due to the nature of the primary MBCRS study.

\section{Conclusion}

The goal of this study was to evaluate the influence of breast-feeding promotion strategies on the rate of breastfeeding during the first 6 months of life. An understanding of these varied approaches to lactation and breastfeeding provides a starting point for the development of effective recommendations for the use of mother's own milk and promotion of breastfeeding that apply to multiple ethnicities. Our research identified significant difference in the rate of exclusive breastfeeding based on mother's age, mother's occupation and parity. The research highlighted the need to counsel nulliparous women more effectively and address their queries accordingly.

\section{Declarations}

\section{Competing Interests}

The authors have declared that no competing interests exist. Independent consultant has also declared no conflict of interest.

\section{Author Contributions}

The study was conceptualized \& designed by SA, MAH \& SBS. LS oversaw field activity. FS, AR were involved in the data analysis. SA \& BT wrote the first draft. KS, AA, AAA, MAH \& SBS critically reviewed the manuscript. All authors reviewed and approved the manuscript.

\section{Acknowledgment}

We would like to acknowledge the hard work and commitment of the study staff, that made the project a success. We are also grateful to the mothers and their infants who contributed to the study. 


\section{Availability of Data and Materials}

The datasets used for the article and the study is available from the corresponding author on request.

\section{References}

1. UNICEF, United Nations Population \& Bank (2015) United Nas Inter-agency Group for Child Mortality Estimation, UNICEF.

2. WHO (2015) WHO-MCEE estimates for child causes of death, 2000-2015, WHO, Geneva, Switzerland.

3. Kimani-Murage EW, Madise NJ, Fotso JC, Kyobutungi C, Mutua MK, et al. (2011) Patterns and determinants of breastfeeding and complementary feeding practices in urban informal settlements, Nairobi Kenya. BMC Public Health 11: 396.

4. Hanieh S, Ha TT, Simpson JA, Thuy TT, Khuong NC, et al. (2015) Exclusive breast feeding in early infancy reduces the risk of inpatient admission for diarrhea and suspected pneumonia in rural Vietnam: a prospective cohort study. BMC Public Health 15: 1166.

5. Cai X, Wardlaw T, Brown DW (2012) Global trends in exclusive breastfeeding. Int Breastfeed J 7: 1-5.

6. Frank NM, Lynch KF, Uusitalo U, Yang J, Lönnrot M, et al. (2019) The relationship between breastfeeding and reported respiratory and gastrointestinal infection rates in young children. BMC pediatrics 19: 339.

7. Horta BL, Bahl R, Martinés JC, Victora CG, WHO (2007) Evidence on the long-term effects of breastfeeding: systematic review and meta-analyses, WHO, Geneva, Switzerland.

8. Horta BL, Victora CG (2013) Long-term effects of breastfeeding-a systematic review, WHO, Geneva, Switzerland.

9. Jamil M, Khanum A, Omer MT, Hamid M (2018) Knowledge, Attitude and Breastfeeding practices of Mothers of Infants: An Experience from a Tertiary Care Hospital in Lahore. EJNM 1-7.

10. National Institute of Population Studies (NIPS) [Pakistan] and ICF International (2019) Pakistan Demographic and Health Survey 2017-18. National Institute of Population Studies, Pakistan.

11. UNICEF, Government of Pakistan (2019) National Nutrition Survey 2018 - Key Findings Report. UNICEF.

12. Heil A, Gonzalez EL, Hilgenfeld T, Kickingereder P, Bendszus M, et al. (2017) Lateral cephalometric analysis for treatment planning in orthodontics based on MRI compared with radiographs: A feasibility study in children and adolescents. PloS one 12: e0174524.

13. Farrukh H, Basheer F, Jalil J (2013) Factors Causing Exclusive Breast-Feeding Failure in a Pakistani Urban Population. Pakistan Armed Forces Medical Journal 63: 329-33. 14. National Institute of Population Studies (NIPS) [Pakistan] and ICF International. 2013. Pakistan Demographic and Health Survey 2012-13, National Institute of Population Studies, Pakistan.

15. WHO, UNICEF (2003) Global strategy for infant and young child feeding. WHO, Geneva, Switzerland.

16. O'Sullivan A, Farver M, Smilowitz JT (2014) The Influence of Early Infant-Feeding --Practices on the Intestinal Microbiome and Body Composition in Infants. Nutr Metab Insights 8: 1-9.

17. Imdad A, Yakoob MY, Bhutta ZA (2011) Effect of breastfeeding promotion interventions on breastfeeding rates, with special focus on developing countries. BMC Public Health 11: S24.

18. Velusamy V, Premkumar P, Kang G (2017) Exclusive breastfeeding practices among mothers in urban slum settlements: pooled analysis from three prospective birth cohort studies in South India. Int Breastfeed J 12: 35.

19. Bhutta Z, Soofi S, Zaidi S, Habib A, Hussain M (2011) Pakistan National Nutrition Survey, The Aga Khan University, Pakistan.

20. Hackman NM, Schaefer EW, Beiler JS, Rose CM, Paul IM (2015) Breastfeeding outcome comparison by parity. Breastfeed Med 10: 156-62.

21. Lee WT, Wong E, Lui SS, Chan V, Lau J (2007) Decision to breastfeed and early cessation of breastfeeding in infants below 6 months old--a population-based study of 3,204 infants in Hong Kong. Asia Pac J Clin Nutr 16: 163-71.

22. Tadesse F, Alemayehu Y, Shine S, Asresahegn H, Tadesse T (2019) Exclusive breastfeeding and maternal employment among mothers of infants from three to five months old in the Fafan zone, Somali regional state of Ethiopia: a comparative cross-sectional study. BMC Public Health 19: 1015.

23. Tsai SY (2013) Impact of a breastfeeding-friendly workplace on an employed mother's intention to continue breastfeeding after returning to work. Breastfeed Med 8: 210-6.

24. Hirani SA, Karmaliani R (2013b) The experiences of urban, professional women when combining breastfeeding with paid employment in Karachi, Pakistan: a qualitative study. Women and Birth 26: 147-15.

25. Hirani SA, Karmaliani R (2013a) Evidence based workplace interventions to promote breastfeeding practices among Pakistani working mothers. Women Birth 26: 10-16. 26. Tahir NM, Al-Sadat N (2013) Does telephone lactation counselling improve breastfeeding practices? A randomised controlled trial. International journal of nursing studies 50: 16-25. 